\title{
Effects of semaglutide on beta cell function and glycaemic control in participants with type 2 diabetes: a randomised, double-blind, placebo-controlled trial
}

\author{
Christoph Kapitza $^{1}$ - Kirsten Dahl ${ }^{2} \cdot$ Jacob B. Jacobsen $^{2} \cdot$ Mads B. Axelsen $^{2}$. \\ Anne Flint ${ }^{2}$
}

Received: 7 February 2017 / Accepted: 31 March 2017 /Published online: 19 May 2017

(C) The Author(s) 2017. This article is an open access publication

\begin{abstract}
Aims/hypothesis Semaglutide is a glucagon-like peptide-1 analogue in development for the treatment of type 2 diabetes. Its effects on first- and second-phase insulin secretion and other measures of beta cell function and glycaemic control were assessed.

Methods In this single-centre, double-blind, placebo-controlled, parallel-group trial, conducted at the Profil Institut für Stoffwechselforschung, Germany, 75 adult (aged 18-64 years) participants with type 2 diabetes (eligibility: $\mathrm{HbA}_{1 \mathrm{c}}$ of $6.5-9.0 \%$ (47.5-74.9 mmol/mol); BMI $20.0-35.0 \mathrm{~kg} / \mathrm{m}^{2}$; and treatment with diet and exercise and/or metformin monotherapy with a dose unchanged in the 30 days prior to screening) were randomised (1:1) to once-weekly s.c. semaglutide $1.0 \mathrm{mg}$ $(0.25,0.5,1.0 \mathrm{mg}$ escalated) or placebo for 12 weeks. Coprimary endpoints were changes from baseline to end of treatment in the first $\left(\mathrm{AUC}_{0-10 \mathrm{~min}}\right)$ and second $\left(\mathrm{AUC}_{10-120 \mathrm{~min}}\right)$ insulin secretion phases, as measured by the IVGTT. An arginine stimulation test (AST) and a $24 \mathrm{~h}$ meal stimulation test were also conducted. A graded glucose infusion test (GGIT) assessed insulin secretion rate (ISR) in treated participants and a group of untreated healthy participants. Safety endpoints were also assessed.
\end{abstract}

Electronic supplementary material The online version of this article (doi:10.1007/s00125-017-4289-0) contains peer-reviewed but unedited supplementary material, which is available to authorised users.

Christoph Kapitza

christoph.kapitza@profil.com

1 Profil Institut für Stoffwechselforschung $\mathrm{GmbH}$, Hellersbergstrasse 9, 41460 Neuss, Germany

2 Novo Nordisk A/S, Søborg, Denmark
Results In total, 37 participants received semaglutide and 38 received placebo. Following IVGTT, for insulin, both $\mathrm{AUC}_{0-10 \mathrm{~min}}$ and $\mathrm{AUC}_{10-120 \mathrm{~min}}$ were significantly increased with semaglutide (estimated treatment ratio $[95 \% \mathrm{CI}] 3.02[2.53,3.60]$ and 2.10 $[1.86,2.37]$, respectively; $p<0.0001)$. The $24 \mathrm{~h}$ meal test showed reduced fasting, postprandial and overall $\left(\mathrm{AUC}_{0-24 \mathrm{~h}}\right)$ glucose and glucagon responses with semaglutide $(p<0.0001)$. The AST showed that maximal insulin capacity increased following semaglutide treatment. During GGIT, semaglutide significantly increased ISR to levels similar to those in healthy participants. Semaglutide was well tolerated.

Conclusions/interpretation Twelve weeks of once-weekly treatment with semaglutide significantly improved beta cell function and glycaemic control in participants with type 2 diabetes.

Trial registration: ClinicalTrials.gov NCT02212067

Funding: The study was funded by Novo Nordisk A/S.

Keywords Beta cell function - Glycaemic control . Placebo-controlled trial $\cdot$ Semaglutide $\cdot$ Type 2 diabetes

\begin{tabular}{ll}
\multicolumn{2}{l}{ Abbreviations } \\
AE & Adverse event \\
AST & Arginine stimulation test \\
$\mathrm{C}_{\max }$ & Maximum concentration \\
ETR & Estimated treatment ratio \\
GGIT & Graded glucose infusion test \\
GLP-1RA & Glucagon-like peptide-1 receptor agonist \\
HOMA-B & HOMA of beta cell function \\
ISR & Insulin secretion rate \\
MDRD & Modification of Diet in Renal Disease \\
PD & Pharmacodynamic \\
PK & Pharmacokinetic
\end{tabular}


SAE Serious adverse event

TEAE Treatment-emergent adverse event

$t_{\max } \quad$ Time to maximum concentration

\section{Introduction}

Beta cell dysfunction is a key component in the pathophysiology of type 2 diabetes [1]. Both the development and progression of type 2 diabetes are marked by declining beta cell mass and function. The UK Prospective Diabetes Study investigators estimated that $50 \%$ of beta cell function may already be lost by the time of diagnosis, and subsequent glycaemic deterioration has been found to be associated with continued loss of beta cell function [2]. Addressing beta cell function is critical to the durability of diabetes treatments [3]. Glucagon-like peptide-1 receptor agonists (GLP-1RAs) appear to have several beneficial effects on beta cell function, offering individuals with type 2 diabetes the potential for enduring glycaemic control [3]. GLP-1 has been shown to prevent glucolipotoxicity in beta cells, thereby helping to protect and improve their overall function [4].

GLP-1RAs help to restore beta cell sensitivity to elevated blood glucose and improve beta cell function across a number of indices in people with type 2 diabetes [5, 6]. These effects have been illustrated in a sequence of studies with the GLP-1 analogue liraglutide vs placebo. Liraglutide treatment resulted in increased first- and second-phase insulin secretion, increased maximal insulin secretion after arginine infusion [7-9], increased HOMA of beta cell function (HOMA-B), a higher level of C-peptide, and a reduced proinsulin-to-insulin ratio [10]. In another study, liraglutide restored beta cell responsiveness to glucose during a stepwise glucose clamp [11], which is in line with trials showing glucose-dependent insulin secretion [12], decreased glucagon secretion from pancreatic islets $[12,13]$ and delayed gastric emptying $[14,15]$.

Semaglutide (Novo Nordisk, Bagsværd, Denmark) is a GLP1 analogue in development for the once-weekly treatment of type 2 diabetes. It has $94 \%$ structural homology to native human GLP-1, and is based on the same acetylation technology as liraglutide [16]. Important structural modifications make semaglutide more resistant to degradation by dipeptidyl peptidase- 4 and improve binding to albumin [16]. These modifications result in a $t_{1 / 2}$ of approximately 1 week, making semaglutide appropriate for once-weekly s.c. administration, with a fully retained potency [17].

This trial investigated the effects of semaglutide $1.0 \mathrm{mg}$ at steady state (after 12 weeks of once-weekly administration) on different aspects of beta cell function in participants with type 2 diabetes. The trial included four tests that together provide a comprehensive picture of changes in beta cell function during treatment with semaglutide. The primary objective was to evaluate the effects of semaglutide on first- and second- phase insulin secretion. Secondary objectives were to investigate the effects of semaglutide on fasting and postprandial glucose, insulin, C-peptide and glucagon concentrations, and on maximal insulin secretory capacity. The beta cell responsiveness to graded glucose infusion was assessed in participants with type 2 diabetes treated with semaglutide or placebo and in a group of healthy untreated participants. Additionally, the study assessed the pharmacokinetics (PK), safety and tolerability of semaglutide.

\section{Methods}

\section{Trial design}

This was a single-centre, randomised, multiple-dose, doubleblind, placebo-controlled, parallel-group trial in participants with type 2 diabetes. A healthy comparator group was also included specifically to evaluate beta cell responsiveness to graded glucose infusion. The trial was conducted at the Profil Institut für Stoffwechselforschung in Germany and complied with the International Conference on Harmonisation Good Clinical Practice guidelines [18] and the Declaration of Helsinki [19]. All participants provided written informed consent and the trial protocol was approved by German health authorities and local independent ethics committees.

Participants with type 2 diabetes were randomised (1:1) to either once-weekly s.c. semaglutide $1.0 \mathrm{mg}$ or placebo. Participants followed a fixed-dose escalation regimen (Fig. 1). Study medication (semaglutide, Novo Nordisk, or volume-matched placebo) was administered for 12 weeks, followed by a 5 week follow-up period. Healthy participants did not receive any treatment.

Semaglutide and placebo were visually identical and were packed blinded. The clinical study group, investigators and all other personnel and participants, except for staff conducting the treatment allocation, were blinded to the randomisation throughout the trial.

\section{Trial population}

Participants were adults (aged 18-64 years). Additional eligibility criteria for participants with type 2 diabetes included: $\mathrm{HbA}_{1 \mathrm{c}}$ of $6.5-9.0 \%$ (47.5-74.9 mmol/mol); BMI of 20.0 $35.0 \mathrm{~kg} / \mathrm{m}^{2}$; and treatment with diet and exercise and/or metformin monotherapy with a dose unchanged in the 30 days prior to screening. At least 24 participants with type 2 diabetes of each sex were required to be enrolled. Participants in the healthy comparator group were required to have a BMI of $24.0-32.0 \mathrm{~kg} / \mathrm{m}^{2}$ and an $\mathrm{HbA}_{1 \mathrm{c}}<6.5 \%$ (47.5 mmol $\left./ \mathrm{mol}\right)$. At least four healthy participants of each sex were required in the control group. 


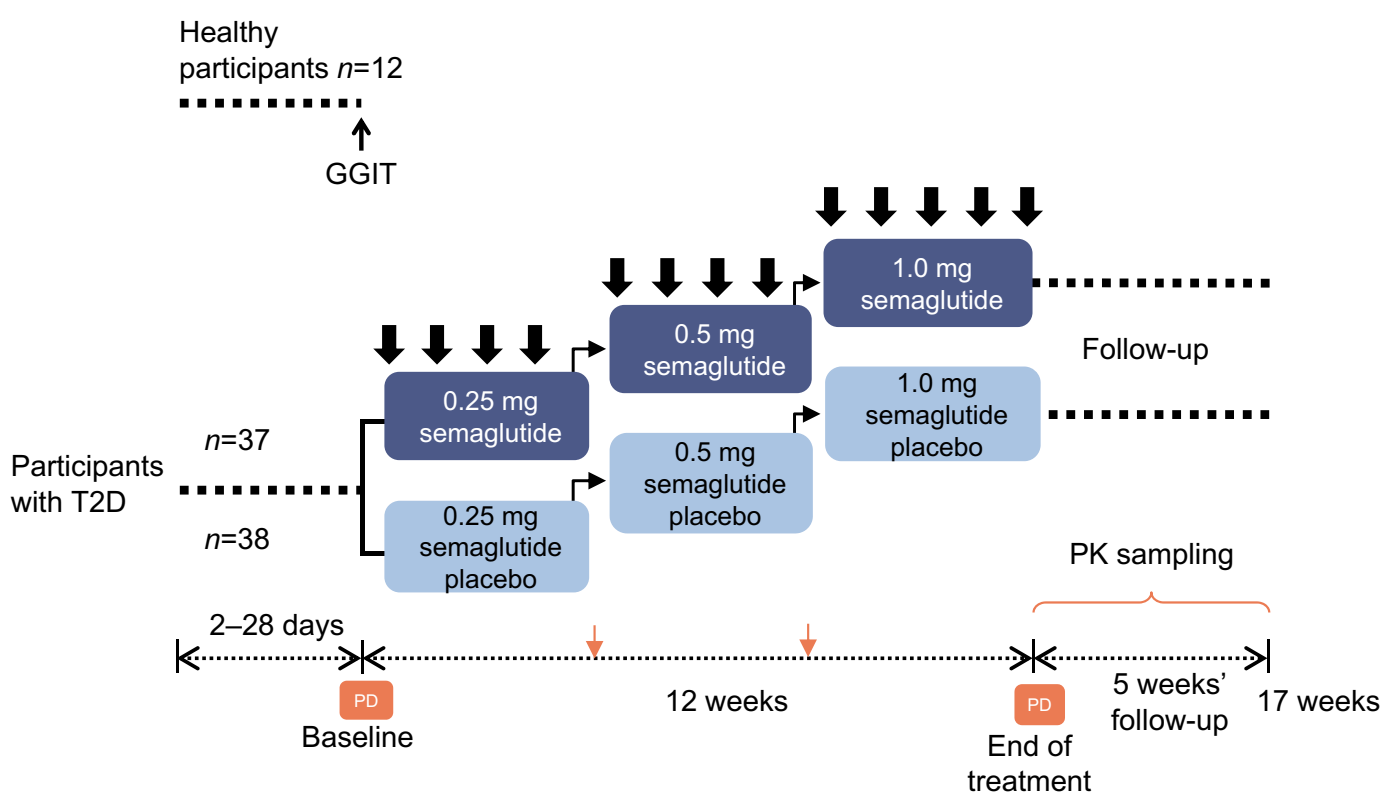

Fig. 1 Trial design. Thick black arrows, study medication dose; thin black arrows, dose escalation; orange arrows, PK sampling; PD assessment comprised: IVGTT, AST, 24 h profiles and GGIT. T2D, type 2 diabetes

Key exclusion criteria for all participants included: any clinically significant disease history or systemic or organ disease; use of any prescription or non-prescription medication that would interfere with trial results, specifically systemic corticosteroids, non-selective beta blockers or thyroid hormones (unless thyroid hormone use was stable 2 months prior to screening).

Additional exclusion criteria for participants with type 2 diabetes included: impaired renal function (eGFR $<60 \mathrm{ml} \mathrm{min}^{-1} 1.73 \mathrm{~m}^{-2}$ per the Modification of Diet in Renal Disease [MDRD] formula); recurrent severe hypoglycaemia; known proliferative retinopathy or maculopathy requiring acute treatment; treatment with glucose-lowering medication other than metformin 3 months prior to screening (except short-term insulin treatment in connection with intercurrent illness).

\section{Randomisation}

Participants were always assigned the lowest available randomisation number. Allocation of treatment was performed by unblinded site staff according to the randomisation list.

\section{Study assessments}

The IVGTT, arginine stimulation test (AST), graded glucose infusion test (GGIT) and meal stimulation over $24 \mathrm{~h}$ were conducted at baseline and end of treatment on different study days (Fig. 1).
The IVGTT evaluated the response to an i.v. bolus injection of $25 \mathrm{~g}$ glucose administered over $2 \mathrm{~min}$. Blood samples for analysis of insulin, C-peptide, glucose and glucagon concentrations were drawn frequently from $30 \mathrm{~min}$ prior to glucose injection through to $2 \mathrm{~h}$ post-injection.

An AST was performed after the completion of the IVGTT to measure maximal insulin secretory capacity. An i.v. glucose infusion $(150 \mathrm{mg} / \mathrm{kg})$ was administered to achieve hyperglycaemia (target $16 \mathrm{mmol} / \mathrm{l}$ ); $2 \mathrm{~h}$ later, $5 \mathrm{~g}$ arginine was administered i.v. over $30 \mathrm{~s}$, and blood samples for the analysis of insulin, C-peptide, glucose and glucagon were drawn frequently until $35 \mathrm{~min}$ after arginine administration.

Fasting and postprandial glucose metabolism were evaluated over a $24 \mathrm{~h}$ period during which three standard meals (breakfast at $0 \mathrm{~h}$, lunch at $5 \mathrm{~h}$, high-protein dinner at $10 \mathrm{~h}$ ) were served. Blood samples were drawn throughout this time to determine $24 \mathrm{~h}$ profiles for glucose, insulin, C-peptide and glucagon.

A GGIT was performed both in participants with type 2 diabetes and healthy participants to assess beta cell responsiveness. The glycaemic start level was $5 \mathrm{mmol} / \mathrm{l}$ (achieved by administration of i.v. glucose or insulin as required). The i.v. glucose infusion was adjusted to achieve sequential plasma glucose targets throughout the $3 \mathrm{~h}$ test of $5,6,7,8,9,10$, 11 and $12 \mathrm{mmol} / \mathrm{l}$, with $25 \mathrm{~min}$ intervals between each target. Blood samples for analysis of insulin, C-peptide, glucose and glucagon levels were drawn throughout the test.

Adverse events (AEs) were recorded from the time of the participant signing informed consent through to the end of follow-up and were regarded as treatment-emergent adverse 
events (TEAEs) if beginning (or increasing in severity) after the first study dose and no later than the end of follow-up.

Blood samples were drawn throughout the trial to assess PK (trough values). Body weight was measured at all visits.

\section{Study endpoints}

In participants with type 2 diabetes The co-primary endpoints were the change from baseline to end of treatment (Week 12) in first- and second-phase insulin secretion, as measured during IVGTT $\left(\mathrm{AUC}_{0-10 \mathrm{~min}}\right.$ and $\mathrm{AUC}_{10-120 \mathrm{~min}}$, respectively).

The secondary IVGTT-related pharmacodynamic (PD) endpoints were the change from baseline to end of treatment in $\mathrm{AUC}_{0-10 \mathrm{~min}}, \mathrm{AUC}_{10-120 \mathrm{~min}}$ and $\mathrm{AUC}_{0-120 \mathrm{~min}}$ with respect to $\mathrm{C}$-peptide, insulin secretion rate (ISR), glucagon and insulin (only $\mathrm{AUC}_{0-120 \mathrm{~min}}$ ).

Secondary PD endpoints measured by the AST included the change from baseline to end of treatment in $\mathrm{AUC}_{0-10 \mathrm{~min}}$ and $\mathrm{AUC}_{0-30 \mathrm{~min}}$ for insulin, ISR and glucagon.

Secondary endpoints measured by meal stimulation included the change from baseline to end of treatment in the $24 \mathrm{~h}$ profiles of plasma glucose, glucagon, serum insulin and C-peptide, measured as total $\mathrm{AUC}_{0-24 \mathrm{~h}}$ during a test day with three standardised meals. Change from baseline to end of treatment in meal-specific AUCs following breakfast, lunch and dinner $(0-5 \mathrm{~h}, 5-10 \mathrm{~h}$ and $10-15 \mathrm{~h}$, respectively) and the corresponding mean postprandial increments were added post hoc.

The change from baseline to end of treatment in body weight was an additional secondary endpoint.

Safety endpoints from baseline to follow-up included the number of TEAEs, the number of severe hypoglycaemic events (as defined by the ADA [20]) or blood glucoseconfirmed (<3.1 mmol/l) hypoglycaemic events, and change from baseline to end of treatment in vital signs and standard laboratory test results.

\section{In participants with type $\mathbf{2}$ diabetes and healthy participants} Graded glucose infusion-related secondary endpoints were change from baseline to end of treatment in the AUC of ISR and glucagon over the 5-12 mmol/l glucose level ( $\mathrm{AUC}_{5-12 \mathrm{mmol}, \mathrm{ISR}}$ and $\mathrm{AUC}_{5-12 \mathrm{mmol} \text {,glucagon, }}$ respectively), and in the slope of the ISR vs glucose curve (dose-response relationship).

\section{PK endpoints}

PK endpoints $\left(\mathrm{AUC}_{0-168}\right.$, maximum concentration $\left[\mathrm{C}_{\max }\right]$, time to maximum concentration $\left[\mathrm{t}_{\max }\right], t_{1 / 2}$ ) were assessed for semaglutide $1.0 \mathrm{mg}$ at steady state, based on blood sampled up to $840 \mathrm{~h}$ after the last dose. Additionally, trough semaglutide concentrations for each dose level at steady state $\left(\mathrm{C}_{\text {trough, }} \mathrm{SS}\right)$ were derived from blood samples drawn 1 week after the fourth dose at each of the three dose levels. Further information on PK endpoints is provided in the electronic supplementary material (ESM).

\section{Statistical analysis}

Sample size was calculated based on the within-participant differences in $\mathrm{AUC}_{0-17 m i n, \text { insulin }}$ and $\mathrm{AUC}_{90-120 \text { min,insulin from }}$ an IVGTT in a crossover liraglutide study (data not published). A dropout rate of 13-15\% was assumed. The enrolment of 37 participants in each treatment group was calculated to provide a combined power of $80.6 \%$ to detect a treatment difference corresponding to $20 \mathrm{nmol} \mathrm{1}^{-1} \mathrm{~h}^{-1}$ and $650 \mathrm{nmol} \mathrm{l}^{-1} \mathrm{~h}^{-1}$ for the first and second insulin secretion phases, respectively, assuming a corresponding SD of $23 \mathrm{nmol} \mathrm{l}^{-1} \mathrm{~h}^{-1}$ and $830 \mathrm{nmol} \mathrm{l}^{-1} \mathrm{~h}^{-1}$.

All AUCs were calculated by non-compartmental modelfree methods using the linear trapezoidal method on the observations and actual time points.

The co-primary endpoints were analysed separately using linear normal models on $\log _{10}$-transformed data, including treatment as a fixed effect and baseline value as a covariate. Estimated treatment difference (ETD) was presented on the original scale as estimated treatment ratio (ETR) with $95 \%$ CIs and $p$ value testing the null hypothesis of no treatment difference.

For all secondary AUC endpoints, the data were analysed and presented in the same way as for the primary endpoints.

The ISR was calculated from population-based C-peptide kinetic variables [21], using ISEC software (version 3.4a, September 1994), in which the insulin grid was set to equal the sample regimen, the basal measurement of $\mathrm{C}$-peptide was included in the calculation and the error of C-peptide measurements was set to 5\% [22]. The ISEC software can be obtained from the developer: Roman Hovorka, PhD, University of Cambridge, UK (https://www.scribd.com/document/ 177600095/Isec-Manual).

For the GGIT, the slope of the ISR vs glucose curve was calculated for each individual profile by a simple regression model on the $\log _{10}$-transformed data. Change from baseline was analysed in the same way as for the primary endpoints.

The mean postprandial increment was calculated as the incremental AUC (the total AUC-time curve minus the area under the pre-meal concentration during the same time interval) divided by the length of the time interval. The change from baseline to end of treatment in mean postprandial increment was analysed in a linear normal model on the original scale with treatment as a fixed effect and baseline value as a covariate.

Change from baseline in body weight was analysed using a mixed model for repeated measurements using all postbaseline data as the dependent variable, and with treatment, 
Table 1 Demographics and baseline characteristics of study populations

\begin{tabular}{lccc}
\hline & Semaglutide & Placebo & Healthy \\
& $1.0 \mathrm{mg}$ & $n=38$ & $n=12$ \\
& $n=37$ & & \\
\hline Age, years & $56(45-64)$ & $57(44-64)$ & $43(24-58)$ \\
$\mathrm{HbA}_{1 \mathrm{c}}, \%$ & $7.3(6.4-8.9)$ & $7.3(5.9-9.0)$ & $\mathrm{N} / \mathrm{A}$ \\
$\mathrm{HbA}_{1 \mathrm{c}}, \mathrm{mmol} / \mathrm{l}$ & $56.1(46.5-73.8)$ & $55.7(41.0-74.9)$ & $\mathrm{N} / \mathrm{A}$ \\
Diabetes duration, years & $8.3(1.0-22.2)$ & $8.7(1.4-21.3)$ & N/A \\
Body weight, kg & $93.2(61.9-119.6)$ & $90.0(64.1-120.0)$ & $26.8(24.5-29.7)$ \\
BMI, kg/m & $29.7(20.7-35.1)$ & $29.7(21.9-35.3)$ & N/A \\
Metformin use, $n(\%)$ & $33(89.1)$ & $34(89.5)$ & $8(66.7)$ \\
Male, $n(\%)$ & $27(73.0)$ & $24(63.2)$ & \\
\hline
\end{tabular}

Data presented are mean (range) unless stated otherwise

N/A, not applicable

visit and treatment nested within visit as factors, and baseline body weight as a covariate. An unstructured covariance matrix was used to describe the variability for the repeated measurements for a participant.

\section{Results}

The trial was conducted between 11 August 2014 and 11 May 2015. In total, 37 participants received semaglutide and 38 participants received placebo (ESM Fig. 1). Baseline characteristics were similar between the semaglutide and placebo groups (Table 1). All participants were white. Two participants receiving semaglutide withdrew from the trial, one due to a serious adverse event (SAE) during the follow-up period and one for other reasons in the middle of the treatment period (ESM Fig. 1). One participant receiving placebo discontinued as a result of a protocol violation prior to end of treatment.

The geometric mean concentration-time profiles for insulin in participants with type 2 diabetes after an i.v. bolus injection of glucose are presented in Fig. 2. First-phase $\left(\mathrm{AUC}_{0-10 \mathrm{~min} \text {,insulin }}\right)$ and second-phase $\left(\mathrm{AUC}_{10-120 \mathrm{~min} \text {,insulin }}\right)$ insulin responses in the IVGTT were significantly increased after 12 weeks of treatment with semaglutide compared with placebo (ETR [95\% CI] 3.02 $[2.53,3.60]$ and $2.10[1.86,2.37]$, respectively, both $p<0.0001$; Fig. 3).

Changes from baseline to end of treatment in the AUC of ISR, C-peptide and glucagon in the IVGTT were also significantly greater in the semaglutide group compared with the placebo group (Fig. 3). The increases in the AUC of insulin and ISR were larger in the first phase than in the second phase of insulin secretion. The treatment effects of semaglutide in increasing the AUC of C-peptide and decreasing the AUC of glucagon were similar in each insulin secretion phase (Fig. 3). The magnitude of change in ISR was reflected in the reduction in glucose during the IVGTT (data not shown).
Observed insulin levels during induced hyperglycaemic conditions immediately prior to the administration of arginine at end of treatment were higher for the semaglutide group than for the placebo group (Fig. 4). The insulin response to arginine was significantly larger after semaglutide treatment compared with placebo (ETR [95\% CI]: $\mathrm{AUC}_{0-10 \mathrm{~min}} 2.82$ [2.39, 3.32], $\mathrm{AUC}_{0-30 \min } 4.42$ [3.74, 5.22]; $p<0.0001$ for both). A larger ISR response was also observed after semaglutide treatment vs placebo (ETR [95\% CI]: $\mathrm{AUC}_{0-10 \min } 1.69$ [1.49, 1.92], $\mathrm{AUC}_{0-30 \mathrm{~min}} 2.69$ [2.38, 3.05]; $p<0.0001$ for both). The glucagon response to arginine was significantly lower with semaglutide treatment vs placebo (ETR $[95 \% \mathrm{CI}]$ : $\mathrm{AUC}_{0-10 \mathrm{~min}}$ 0.80 [0.75, 0.87], $\mathrm{AUC}_{0-30 \min } 0.82$ [0.78, 0.87]; $p<0.0001$ for both). As can be seen from both the $\mathrm{AUC}_{0-10 \mathrm{~min}}$ and the $\mathrm{AUC}_{0-30 \mathrm{~min}}$ results, these differences between treatment groups occurred throughout the duration of the AST.

In the $24 \mathrm{~h}$ meal test, compared with placebo, $\mathrm{AUC}_{0-24 \mathrm{~h}}$ for glucose and glucagon was significantly reduced after semaglutide treatment (ETR [95\% CI]: 0.78 [0.74, 0.82] and

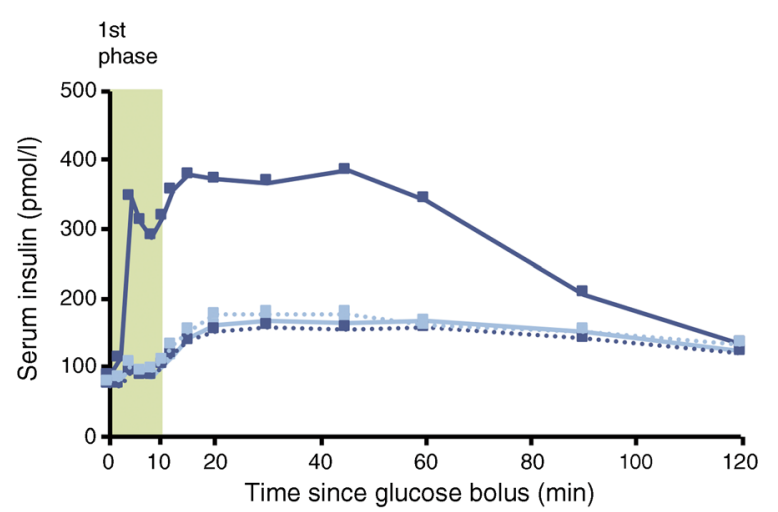

Fig. 2 Geometric mean insulin response to an IVGTT in participants with type 2 diabetes before and after 12 weeks of treatment with semaglutide ( $n=37$, dark blue) or placebo ( $n=37$, light blue). One participant who received an incorrect glucose dose was excluded from all IVGTT analyses. Dotted lines represent baseline values and solid lines represent end of treatment values 
Fig. 3 ETR

(semaglutide:placebo) for endpoints from an IVGTT. Endpoints are the change from baseline to end of treatment in the AUC. One participant who received an incorrect glucose dose was excluded ( $n=36$ in both groups). 0-10 min, first phase; 10-120 min, second phase. All endpoints listed are change from baseline to end of treatment in AUC. All $p$ values $\leq 0.0002$

\begin{tabular}{|c|c|c|c|c|c|}
\hline Endpoint & Time (min) & & & ETR & $(95 \%$ CI $)$ \\
\hline Insulin & $0-10$ & 1 & $\longmapsto$ & 3.02 & $(2.53,3.60)$ \\
\hline Insulin & $10-120$ & i & $\longmapsto$ & 2.10 & $(1.86,2.37)$ \\
\hline Insulin & $0-120$ & 1 & $1=$ & 2.16 & $(1.91,2.43)$ \\
\hline ISR & $0-10$ & re & $\longmapsto$ & 2.93 & $(2.50,3.43)$ \\
\hline ISR & $10-120$ & I & $\longmapsto$ & 1.75 & $(1.60,1.91)$ \\
\hline ISR & $0-120$ & 1 & $1=-1$ & 1.83 & $(1.68,1.99)$ \\
\hline C-peptide & $0-10$ & 1 & $\longmapsto$ & 1.73 & $(1.59,1.88)$ \\
\hline C-peptide & $10-120$ & I & $\mapsto$ & 1.74 & $(1.61,1.87)$ \\
\hline C-peptide & $0-120$ & 1 & 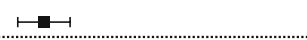 & 1.74 & $(1.62,1.87)$ \\
\hline Glucagon & $0-10$ & $1-11$ & & 0.90 & $(0.85,0.96)$ \\
\hline Glucagon & $10-120$ & $1-1$ & & 0.90 & $(0.85,0.95)$ \\
\hline \multirow[t]{3}{*}{ Glucagon } & $0-120$ & $1=1$ & & 0.90 & $(0.85,0.95)$ \\
\hline & & $\cdots$ & & & \\
\hline & 0.5 & 1.0 & 2.0 & & \\
\hline
\end{tabular}

respectively; $p<0.0001$ for both). Changes in glucagon levels with semaglutide treatment $\left(\mathrm{AUC}_{5-12 \mathrm{mmol}}\right)$ were significantly lower compared with placebo $(0.87[0.82,0.93] ; p<0.0001)$.

The end of treatment values of these variables $\left(\mathrm{AUC}_{5-12 \mathrm{mmol}}\right.$ for ISR, glucagon and the slope of the ISR vs glucose curve) in the semaglutide group were more similar to those for the healthy participants than for the participants receiving placebo, especially for the ISR and the slope of the ISR-glucose concentration curve (Table 3, Fig. 6).

Body weight decreased by $4.2 \mathrm{~kg}$ in the semaglutide group, compared with $0.1 \mathrm{~kg}$ for placebo (ETD [95\% CI] $-4.1[-5.1,-3.1])$ over 12 weeks.

The PK data for semaglutide were consistent with those previously reported. PK values, including trough values during dose escalation, were indicative of treatment compliance (ESM Fig. 2 and ESM Table 1).

TEAEs were reported in $75.7 \%$ of participants receiving semaglutide and $55.3 \%$ receiving placebo (ESM Table 2). The most common type of TEAE in the semaglutide group, and thus the primary reason for the difference in incidence of

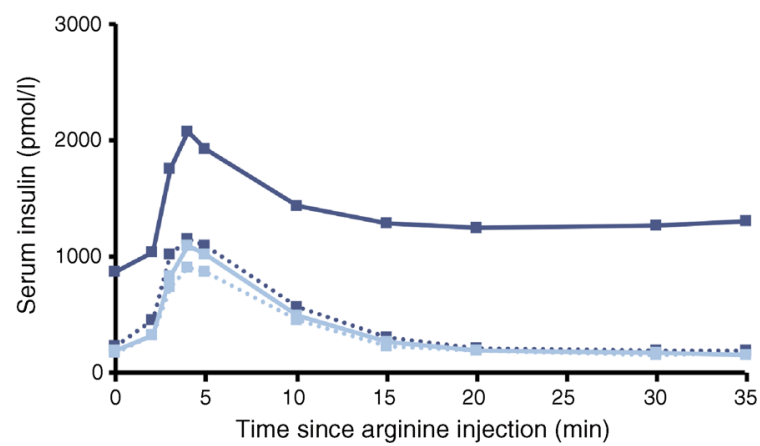

Fig. 4 Serum insulin over time following hyperglycaemic ASTs in participants with type 2 diabetes before and after 12 weeks of treatment with semaglutide ( $n=37$, dark blue) or placebo ( $n=38$, light blue). Dotted lines represent baseline values and solid lines represent end of treatment values 
Table 2 Meal stimulation test. Semaglutide: placebo differences in the change from baseline to end of treatment AUC for glucose, glucagon, insulin and C-peptide, shown as absolute fasting and postprandial AUC, and mean postprandial increments

\begin{tabular}{|c|c|c|c|c|c|}
\hline & Fasting state & Breakfast & Lunch & Dinner & Overall \\
\hline \multicolumn{6}{|l|}{ Absolute fasting and postprandial } \\
\hline Glucose & $0.78(0.74,0.83)$ & $0.71(0.67,0.76)$ & $0.79(0.74,0.85)$ & $0.80(0.75,0.86)$ & $0.78(0.74,0.82)$ \\
\hline Glucagon & $0.92(0.86,0.99)$ & $0.86(0.82,0.91)$ & $0.86(0.81,0.91)$ & $0.85(0.79,0.91)$ & $0.88(0.83,0.93)$ \\
\hline Insulin & $1.30(1.11,1.53)$ & $0.95(0.84,1.07)$ & $1.05(0.94,1.18)$ & $0.90(0.81,1.01)$ & $1.01(0.93,1.10)$ \\
\hline C-peptide & $1.23(1.14,1.32)$ & $1.03(0.96,1.10)$ & $1.06(0.99,1.14)$ & $0.97(0.91,1.04)$ & $1.05(1.00,1.10)$ \\
\hline \multicolumn{6}{|l|}{ Mean postprandial increment, } \\
\hline ETD $(95 \%$ CI $)$ & - & $\mathrm{iAUC}_{0-5 \mathrm{~h}} / 5 \mathrm{~h}$ & $\mathrm{iAUC}_{5-10 \mathrm{~h}} / 5 \mathrm{~h}$ & iAUC $_{10-15 \mathrm{~h}} / 5 \mathrm{~h}$ & - \\
\hline Glucose, mmol/1 & N/A & $-1.11(-1.52,-0.71)$ & $-0.92(-1.30,-0.54)$ & $-0.60(-0.93,-0.28)$ & N/A \\
\hline Glucagon, ng/l & N/A & $-12.86(-23.38,-2.34)$ & $-5.82(-13.86,2.23)$ & $-11.49(-21.91,-1.06)$ & N/A \\
\hline Insulin, $\mathrm{pmol} / 1$ & N/A & $-43.47(-73.06,-13.89)$ & $-15.33(-50.80,20.15)$ & $-42.90(-81.81,-3.99)$ & N/A \\
\hline C-peptide, nmol/1 & N/A & $-0.192(-0.316,-0.067)$ & $-0.086(-0.229,0.056)$ & $-0.275(-0.423,-0.128)$ & N/A \\
\hline
\end{tabular}

iAUC, incremental AUC; N/A, not applicable

TEAEs between the treatment groups, was gastrointestinal, reported in $19(51.4 \%)$ participants in the semaglutide group and six $(15.8 \%)$ participants in the placebo group. Of these, the most common events were nausea, vomiting and diarrhoea, reported in seven $(18.9 \%)$, six $(16.2 \%)$ and four $(10.8 \%)$ semaglutide-treated participants, respectively, and in two $(5.3 \%)$, two $(5.3 \%)$ and three $(7.9 \%)$ placebo-treated participants, respectively. Most AEs were mild to moderate in severity and transient in nature. One TEAE in the semaglutide group with a fatal outcome (pedestrian traffic accident) led to participant withdrawal. The investigator assessed the event as unlikely to be related to treatment. Two other TEAEs classed as serious occurred: one instance of angina pectoris and one hypertensive emergency. In both instances, the event resolved and the participant recovered.

\section{Discussion}

This study investigated the effects of 12 weeks of once-weekly s.c. semaglutide treatment on various aspects of beta cell function in participants with type 2 diabetes. It was demonstrated by IVGTT that semaglutide treatment increased first- and secondphase insulin secretion threefold and twofold, respectively, compared with placebo. Correspondingly, levels of glucagon and glucose were decreased with semaglutide vs placebo. Similar findings have been reported for once-daily liraglutide, which significantly increased both first- and second-phase insulin secretion after 14 weeks of treatment in participants with type 2 diabetes [7].

Results from the AST under hyperglycaemic conditions showed that maximal insulin capacity had improved following semaglutide treatment. Despite insulin levels prior to the test being higher in semaglutide-treated participants than in participants receiving placebo, insulin levels increased immediately in response to the stimulus and remained high for the duration of the test. This effect could contribute to the reported efficacy of semaglutide in improving glycaemic control [23], particularly as recent research suggests that individuals with sustained endogenous insulin-secreting capacity may benefit more from GLP-1RA therapy [24].

In the $24 \mathrm{~h}$ meal test, encompassing three standardised meals, semaglutide reduced glucose and glucagon, and increased C-peptide levels (a marker of endogenous insulin production) in comparison with placebo. Reductions in glucose and glucagon levels with semaglutide were observed in the fasting as well as in the postprandial state, both in terms of the absolute levels and the incremental response. As shown with a

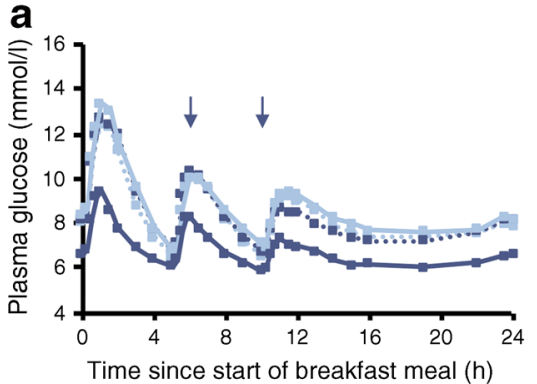

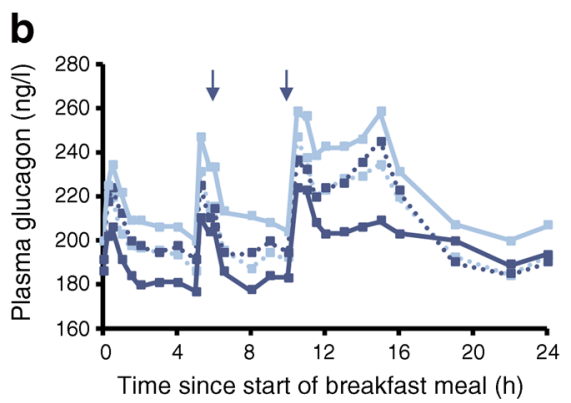

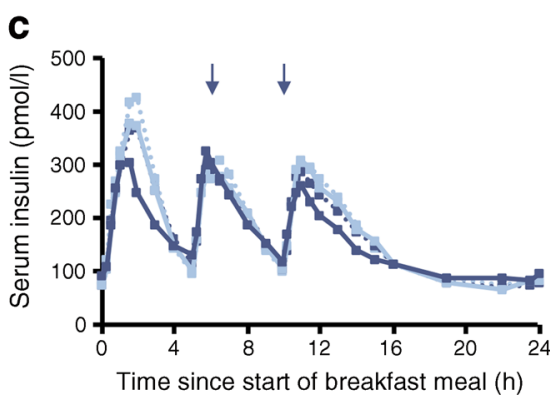

Fig. 5 Plasma glucose (a), plasma glucagon (b) and serum insulin (c) $24 \mathrm{~h}$ profiles in participants with type 2 diabetes before and after receiving 12 weeks of treatment with semaglutide ( $n=37$, dark blue) or placebo

( $n=38$, light blue). Dotted lines represent baseline values and solid lines represent end of treatment values. Arrows represent the start of meals 
Table 3 Endpoints from GGIT test after 12 weeks of treatment

\begin{tabular}{|c|c|c|c|}
\hline & $\begin{array}{l}\text { Semaglutide } 1.0 \mathrm{mg} \\
n=36^{\mathrm{a}}\end{array}$ & $\begin{array}{l}\text { Placebo } \\
n=37^{\mathrm{b}}\end{array}$ & $\begin{array}{l}\text { Healthy participants, } \\
\text { no treatment } \\
n=12\end{array}$ \\
\hline $\mathrm{ISR}_{\mathrm{AUC}} \mathrm{AL}_{-12 \mathrm{mmol}}, \mathrm{pmol} / \mathrm{kg}$ & $43.9(44.6)$ & $19.5(43.3)$ & $45.7(31.0)$ \\
\hline $\begin{array}{l}\text { Slope ISR vs glucose, } \text { pmol } \times \\
1 /\left(\operatorname{min~} \mathrm{mol}^{-1} \mathrm{~kg}^{-1}\right)\end{array}$ & $1.3(53.7)$ & $0.6(41.2)$ & $1.4(35.8)$ \\
\hline Glucagon $\mathrm{AUC}_{5-12 \mathrm{mmol}}, \mathrm{ng} / \mathrm{l}$ & $994.9(17.4)$ & $1129.4(19.2)$ & $875.9(12.9)$ \\
\hline
\end{tabular}

Data are presented as geometric mean (coefficient of variance, \%)

a One participant was withdrawn for 'other reason' prior to the end of treatment visit and was not included in this analysis

${ }^{\mathrm{b}}$ One participant discontinued prior to the end of treatment visit and was not included in this analysis

other GLP-1RAs, a higher insulin level was observed in the fasting state after semaglutide treatment despite lower fasting glucose levels, possibly due to the well-known incretin effect of the GLP-1 class [3, 15]. Notwithstanding this higher initial insulin level, similar postprandial insulin profiles were observed with semaglutide treatment and placebo. The similar insulin profiles were probably related to the lower incremental increase in glucose. A potential additional factor explaining this observation may be the loss of body weight during the course of the trial, possibly impacting insulin resistance. These findings are consistent with previous observations on the relative contributions of insulin stimulation and glucagon inhibition in overall glucose turnover mediated by exogenous GLP-1 and other GLP-1RAs [25]. Mean $2 \mathrm{~h}$ postprandial glucose was lower than the $2 \mathrm{~h}$ postprandial goal $(<10 \mathrm{mmol} / \mathrm{l})$ as recommended by the ADA [26], and more than $80 \%$ of the semaglutide-treated participants achieved the ADA target.

Beta cell responsiveness was markedly increased after treatment with semaglutide compared with placebo, and at

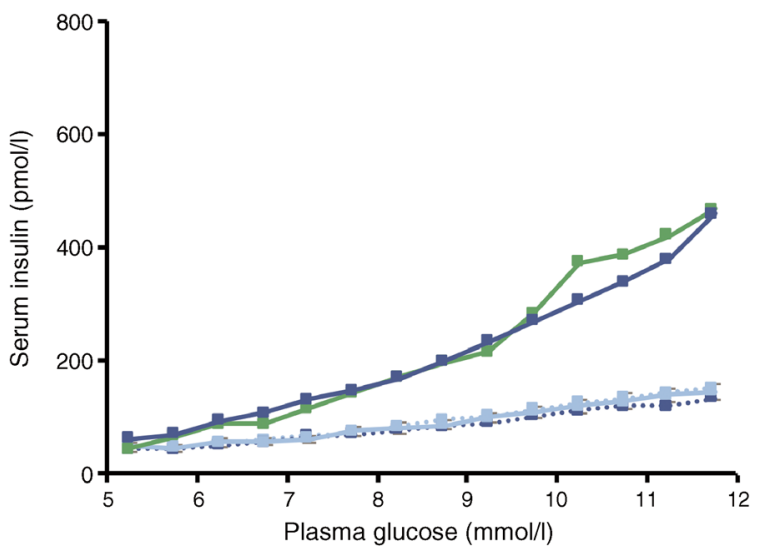

Fig. 6 Insulin response to a graded glucose infusion in participants with type 2 diabetes before and after receiving 12 weeks of treatment with semaglutide ( $n=37$, dark blue) or placebo ( $n=38$, light blue), and in healthy participants ( $n=12$, green). Dotted lines represent baseline values and solid lines represent end of treatment values the end of treatment closely resembled that of healthy participants, as measured by the GGIT. It is important to note, however, that BMI was higher in participants with type 2 diabetes than in healthy participants; differences in fat mass could potentially influence insulin sensitivity and this should be kept in mind when interpreting these results [27]. In addition, mean age was also higher in participants with type 2 diabetes than in healthy participants; the negative impact of ageing on beta cell responsiveness, triacylglycerol levels [28] and glucose homeostasis should also be considered in this context [29]. The possible role of managing beta cell function in type 2 diabetes, as a means to delay disease progression, has been a subject of discussion among researchers [30]. GLP-1RAs have attenuated beta cell dysfunction to varying degrees both in animal models [31] and in humans with type 2 diabetes [32].

Semaglutide treatment was associated with marked weight loss, even during this relatively short trial. These weight loss effects have persisted in trials of longer durations with semaglutide [33] and other GLP-1RAs [34, 35]. The high proportion of individuals with type 2 diabetes who are overweight or obese makes the combination of glycaemic control and body weight loss highly relevant to clinical practice [36].

No new safety or tolerability issues were observed for semaglutide. Most AEs were mild or moderate in severity and there were no serious TEAEs that were considered likely to be related to treatment. Longer and larger scale clinical trials will provide more information on the safety profile of semaglutide [23]. As reported with other GLP-1RAs [37, 38], gastrointestinal disorders were the most commonly reported AEs with semaglutide treatment. The incidence of such events has been shown to diminish over time with liraglutide $[38,39]$ and can be partially ameliorated with dose escalation at the start of treatment [17].

This study was limited by its relatively small size and short duration. The SUSTAIN clinical trial programme, comprising six global phase 3 trials, will determine how the improvements in beta cell function with semaglutide translate into clinical benefits, compared with current treatment options. 
In conclusion, the results of the current study are consistent with previous findings during treatment with the GLP-1 analogue liraglutide [25], and suggest that treatment with semaglutide may offer a protective effect on beta cell function. In addition, the results show that semaglutide is a promising once-weekly GLP-1 analogue for the treatment of type 2 diabetes, associated with improved beta cell responsiveness comparable with that observed in healthy individuals. All aspects of beta cell function, including first- and second-phase insulin responses, were significantly increased in participants with type 2 diabetes treated with semaglutide vs placebo after 12 weeks of treatment. In addition, semaglutide reduced fasting and postprandial glucose and glucagon levels, compared with placebo, in a $24 \mathrm{~h}$ meal test. Improved glycaemic responses appeared to be a combined effect on the pancreas of increased insulin secretion and decreased glucagon response. Semaglutide was well tolerated.

Acknowledgements We thank L. Langley and I. Seymour (AXON Communications, London, UK) for medical writing and editorial assistance. All contributors received compensation from Novo Nordisk A/S. Some of the data were presented as an abstract at the 52nd EASD Annual Meeting in Munich in 2016.

Data availability Researchers can be granted access to the anonymised data generated in this study. Access can be requested via www. novonordisk-trials.com/website/content/how-to-access-clinical-trialdatasets.aspx. The request for access will be reviewed and authorised by an independent review board.

Funding This study was funded by an unrestricted grant from Novo Nordisk A/S.

Duality of interest $\mathrm{CK}$ is an employee and co-owner of Profil, which has received research funds from Adocia, Astra Zeneca, Biocon, Boehringer Ingelheim, Dance Pharmaceuticals, Eli Lilly, Gulf Pharmaceutical Industries, Johnson \& Johnson, Marvel Life Sciences Ltd., Medtronic, Medimmune, Novartis, Novo Nordisk, Roche Diagnostics, Sanofi, Senseonics and Zealand Pharma. CK has received advisory and speaker fees from Sanofi, and travel grants from Novo Nordisk and Sanofi. MBA, KD, AF and JBJ are full-time employees of Novo Nordisk A/S.

Author contribution $\mathrm{AF}$ and $\mathrm{CK}$ designed the study. MBA, KD, AF, $\mathrm{CK}$ and JBJ participated in the conduct of the study, data collection, data analysis and interpretation, and writing and revision of the manuscript. All authors gave approval of the final version of the manuscript for publication. $\mathrm{CK}$ is the guarantor of this work.

Open Access This article is distributed under the terms of the Creative Commons Attribution 4.0 International License (http:// creativecommons.org/licenses/by/4.0/), which permits unrestricted use, distribution, and reproduction in any medium, provided you give appropriate credit to the original author(s) and the source, provide a link to the Creative Commons license, and indicate if changes were made.

\section{References}

1. Halban PA, Polonsky KS, Bowden DW et al (2014) Beta-cell failure in type 2 diabetes: postulated mechanisms and prospects for prevention and treatment. Diabetes Care 37:1751-1758

2. UK Prospective Diabetes Study Group (1995) U.K. Prospective Diabetes Study 16. Overview of 6 years' therapy of type II diabetes: a progressive disease. Diabetes 44:1249-1258

3. Garber AJ (2011) Incretin effects on $\beta$-cell function, replication, and mass: the human perspective. Diabetes Care 34:S258-S263

4. Portha B, Tourrel-Cuzin C, Movassat J (2011) Activation of the GLP-1 receptor signalling pathway: a relevant strategy to repair a deficient beta-cell mass. Exp Diabetes Res 2011:376509

5. Wysham C, Blevins T, Arakaki R et al (2014) Efficacy and safety of dulaglutide added onto pioglitazone and metformin versus exenatide in type 2 diabetes in a randomized controlled trial (AWARD-1). Diabetes Care 37:2159-2167

6. Ahrén B, Leguizamo Dimas A, Miossec P, Saubadu S, Aronson R (2013) Efficacy and safety of lixisenatide once-daily morning or evening injections in type 2 diabetes inadequately controlled on metformin (GetGoal-M). Diabetes Care 36:2543-2550

7. Vilsbøll T, Brock B, Perrild H et al (2008) Liraglutide, a once-daily human GLP-1 analogue, improves pancreatic B cell function and arginine-stimulated insulin secretion during hyperglycaemia in patients with type 2 diabetes mellitus. Diabet Med 25:152-156

8. Kondo Y, Satoh S, Osada UN, Terauchi Y (2015) Early liraglutide treatment improves beta-cell function in patients with type 2 diabetes: a retrospective cohort study. Endocr J 62:971-980

9. Degn KB, Juhl CB, Sturis J et al (2004) One week's treatment with the long-acting glucagon-like peptide 1 derivative liraglutide (NN2211) markedly improves 24-h glycemia and alpha- and betacell function and reduces endogenous glucose release in patients with type 2 diabetes. Diabetes 53:1187-1194

10. Zinman B, Gerich J, Buse JB et al (2009) Efficacy and safety of the human glucagon-like peptide-1 analog liraglutide in combination with metformin and thiazolidinedione in patients with type 2 diabetes (LEAD-4 Met+TZD). Diabetes Care 32:1224-1230

11. Chang AM, Jakobsen G, Sturis J et al (2003) The GLP-1 derivative NN2211 restores beta-cell sensitivity to glucose in type 2 diabetic patients after a single dose. Diabetes 52:1786-1791

12. Hare KJ, Vilsbøll T, Asmar M, Deacon CF, Knop FK, Holst JJ (2010) The glucagonostatic and insulinotropic effects of glucagon-like peptide 1 contribute equally to its glucose-lowering action. Diabetes 59:1765-1770

13. Flint A, Raben A, Astrup A, Holst JJ (1998) Glucagon-like peptide 1 promotes satiety and suppresses energy intake in humans. J Clin Invest 101:515-520

14. Hjerpsted J, Brooks A, Flint A, Kvist T, Blundell J (2016) Semaglutide improves postprandial glucose and lipid metabolism and delays first-hour gastric emptying in subjects with obesity. American Diabetes Association 76th Scientific Sessions, New Orleans, LA, USA, 10-14 June, 2016. Abstract 1046-P

15. Marathe CS, Rayner CK, Jones KL, Horowitz M (2013) Relationships between gastric emptying, postprandial glycemia, and incretin hormones. Diabetes Care 36:1396-1405

16. Lau J, Bloch P, Schaffer L et al (2015) Discovery of the once-weekly glucagon-like peptide-1 (GLP-1) analogue semaglutide. J Med Chem 58:7370-7380

17. Kapitza C, Nosek L, Jensen L, Hartvig H, Jensen CB, Flint A (2015) Semaglutide, a once-weekly human GLP-1 analog, does not reduce the bioavailability of the combined oral contraceptive, ethinylestradiol/levonorgestrel. J Clin Pharmacol 55:497-504

18. European Agency for the Evaluation of Medicinal Products. International Conference on Harmonisation-World Health Organization Guideline for good clinical practice ICH 
Harmonised tripartite guideline good clinical practice www.ema. europa.eu/docs/en_GB/document_library/Scientific_guideline/ 2009/09/WC500002874.pdf. Accessed 10 February 2017

19. World Medical Association (2013) World Medical Association Declaration of Helsinki: ethical principles for medical research involving human subjects. JAMA 310:2191-2194

20. American Diabetes Association Workgroup on Hypoglycemia (2005) Defining and reporting hypoglycemia in diabetes: a report from the American Diabetes Association Workgroup on Hypoglycemia. Diabetes Care 28:1245-1249

21. Van Cauter E, Mestrez F, Sturis J, Polonsky KS (1992) Estimation of insulin secretion rates from C-peptide levels. Comparison of individual and standard kinetic parameters for C-peptide clearance. Diabetes 41:368-377

22. Hovorka R, Soons PA, Young MA (1996) ISEC: a program to calculate insulin secretion. Comput Methods Prog Biomed 50: 253-264

23. Nauck MA, Petrie JR, Sesti G et al (2016) A phase 2, randomized, dose-finding study of the novel once-weekly human GLP-1 analog, semaglutide, compared with placebo and open-label liraglutide in patients with type 2 diabetes. Diabetes Care 39:231-241

24. Kozawa J, Inoue K, Iwamoto R et al (2012) Liraglutide is effective in type 2 diabetic patients with sustained endogenous insulinsecreting capacity. J Diabetes Investig 3:294-297

25. Flint A, Kapitza C, Hindsberger C, Zdravkovic M (2011) The oncedaily human glucagon-like peptide-1 (GLP-1) analog liraglutide improves postprandial glucose levels in type 2 diabetes patients. Adv Ther 28:213-226

26. American Diabetes Association (2016) Standards of medical care in diabetes-2016 abridged for primary care providers. Clin Diabetes $34: 3-21$

27. Ho TP, Zhao X, Courville AB et al (2015) Effects of a 12-month moderate weight loss intervention on insulin sensitivity and inflammation status in nondiabetic overweight and obese subjects. Horm Metab Res 47:289-296

28. Murakata Y, Fujimaki T, Yamada Y (2015) Age-related changes in clinical parameters and their associations with common complex diseases. Biomed Rep 3:767-777
29. De Tata V (2014) Age-related impairment of pancreatic Beta-cell function: pathophysiological and cellular mechanisms. Front Endocrinol (Lausanne) 5:138

30. Standl E (2007) The importance of beta-cell management in type 2 diabetes. Int J Clin Pract 61(Suppl 153):10-19

31. Zheng J, Chen T, Zhu Y et al (2015) Liraglutide prevents fast weight gain and beta-cell dysfunction in male catch-up growth rats. Exp Biol Med (Maywood) 240:1165-1176

32. Tudurí E, López M, Diéguez C, Nadal A, Nogueiras R (2016) Glucagon-like peptide 1 analogs and their effects on pancreatic islets. Trends Endocrinol Metab 27:304-318

33. Ahmann AJ, Capehorn M, Charpentier G et al (2016) Efficacy and safety of once-weekly semaglutide vs exenatide ER after 56 weeks in subjects with type 2 diabetes (SUSTAIN 3). Diabetologia 59:S76 (Abstract)

34. Madsbad S (2016) Review of head-to-head comparisons of glucagon-like peptide-1 receptor agonists. Diabetes Obes Metab 18:317-332

35. Madsbad S, Kielgast U, Asmar M, Deacon CF, Torekov SS, Holst JJ (2011) An overview of once-weekly glucagon-like peptide-1 receptor agonists - available efficacy and safety data and perspectives for the future. Diabetes Obes Metab 13:394-407

36. Daousi C, Casson IF, Gill GV, MacFarlane IA, Wilding JP, Pinkney JH (2006) Prevalence of obesity in type 2 diabetes in secondary care: association with cardiovascular risk factors. Postgrad Med J 82:280-284

37. Diamant M, van Gaal L, Stranks S et al (2010) Once weekly exenatide compared with insulin glargine titrated to target in patients with type 2 diabetes (DURATION-3): an open-label randomised trial. Lancet 375:2234-2243

38. Marre M, Shaw J, Brändle M et al (2009) Liraglutide, a once-daily human GLP-1 analogue, added to a sulphonylurea over 26 weeks produces greater improvements in glycaemic and weight control compared with adding rosiglitazone or placebo in subjects with type 2 diabetes (LEAD-1 SU). Diabet Med 26:268-278

39. Nauck M, Frid A, Hermansen K et al (2009) Efficacy and safety comparison of liraglutide, glimepiride, and placebo, all in combination with metformin, in type 2 diabetes: the LEAD (liraglutide effect and action in diabetes)-2 study. Diabetes Care 32:84-90 